\title{
Zur Axiomatik der Mengenlehre ${ }^{1}$ ).
}

\author{
Von
}

A. Schoenflies in Frankfurt a. M.

Die Hilbertsche Grundlegung der Geometrie darf für alle analogen Untersuchungen als vorbildlich gelten. Zwei ihrer Eigenschaften sind es, auf die es hier ankommt. Erstens wird von allen sprachlichen Definitionen der Objekte, mit denen sie operiert, wie Punkt, Gerade, zwischen usw. abgesehen; nur ihre gegenseitigen Beziehungen und deren Grondgesetze werden axiomatisch an die Spitze gestellt ${ }^{2}$ ). Zweitens werden die Axiome in versohiedene Gruppen gewisser Eigenart und Tragweite gespalten (die des Schneidens und Verbindens, die Axiome der Ordnung, der Kongruenz usw.), und es ist eine wesentliche Aufgabe des axiomatischen Aufbaues, zu prüfen, bis zu welchen Resultaten eine einzelne oder mehrere dieser Gruppen für sich führen. Die gleiche Behandlung eignet sich für die Mengenlehre. Von sprachlicher Einführung der Begriffe Menge, Bereich usw. ist daher ebenso abrusehen, wie von der des Punktes oder Raumes. Ebenso kann man hier gewisse Axiomgruppen unterscheiden, die Axiome der Äquivalenz, die Axiome der Ordnung usw., und kann die gleichen Fragen stellen, wie im Gebiet der Geometrie. Dies soll im folgenden geschehen, und awar für denjenigen Teil, der nur mit der Aquivalenz der Mengen, der Mengenteilung und Mengenverbindung, sowie der Mengenvergleichung operiert.

2) Mit Zusatz abgedrnokt aus Amsterdam Ao. Procoedings 22 (1920).

7) Der Inukbidische Anfban beginnt noah mit den Worten: Hin Pankt ist, was keine Teile hat. tine Linie ist eine Lünge ohne Breite usw. In dem Vergieht auf alle solohen sprachlichen Begriffsbestimmangen liegt einer der wesentlichen durch Hilbert besonders modern gewordenon Gedanken. Ubrigens hatto schon Pasch durch seine Vorlesungen tiber nenere Geometrie (Leiprig, 1882) in dieser Richtrng vorbild: bah gewirkt. Die Mengenlehre hat sich diesem Gedanken bisher nicht erschlossen. 
Will man die Probleme der Mengenlehre einer derartigen Behandlung unterwerfen, so ist es oberstes Erfordernis, die Begriffe der endlichen und der unendlichen Menge auf einer Grundlage einzuführen, die nux die ebengenannten Fundamentalbegriffe benutzt. Solche Definitionen sind $j a$ in der Dedekindschen Begriffsbestimmang vorhanden: Eine Menge $\boldsymbol{M}$ heißt unendlich, wenn es eine (echte) Teilmenge $M^{\prime}$ von $M$ gibt, die äquivalent $M$ ist; sie heißt endlich, wenn es eine solche Teilmenge nicht gibt. Sie haben daher den alleinigen Ausgangspunkt zu bilden.

Die historische Entwicklung der Mengenlehre ist freilich wesentlich anders vor sich gegangen. Während vorstehend die unendliche Menge als das logiseh positiv bestimmte Objekt erscheint, und die endliche Menge als ihr logisches Gegenteil, ist die historische Entwicklung umgekehrt von den endlichen Mengen als wohlbekannten mathematischen Objekten ausgegangen, und hat die unendlichen Mengen als Gegensatz der endlichen Mengen eingeführt. Der so benutzte Begriff der endlichen Menge gehört aber bereits einem Gebiet an, das isich nicht mehr ansschließlich an die Äquivalenzberiehungen ansehlieBt, Der historisch überkommene Begriff der endlichen Menge ruht ja überhaupt nicht auf axiomatischer Grundlage. Mag man inn sprachlich oder empirisch oder anschaulich auffassen, ex war im wesentlichen an der Hand des Zahlbegriffs entstanden und ruht jedenfalls auf Voraussetzungen, in die auch die Ordnung als Grundbegriff eingeht. Diese gehört aber bereits einer Begriffsgruppe an, von der hier abzusehen ist. So laufen in der historischen Entwicklung der Mengenlehre zwei wesentlich verschiedene Bestimmungen der endlichen und unendlichen Mengen nnvermittelt nebeneinander her and exschweren infolgedessen die Frage nach dem, was den einzelnen Sätzen axiomatisch zugrunde liegt. Auch insofern ist eine Klärung des Sechverhalts wïnschenswert.

Das Resultat erweist sich in zwei Punkten als durchaus eigenartig. Die Vergleichung der Mengen bezüglich ihres Größencharakters ist nämlich nichts, was dem Mengenbegriff allein eigentümlich ist; sie betrifft allgemeiner alle Objekte, für die man das Ganze und den Bestandteil unterseheiden kann. Die Axiomatik, die hier entwickelt wird, ist also richtiger eine Axiomatiz der Gröpenlehre, and zwar in dem besonderen Fall, das es auch GröBen von unendlichem Charakter gibt. Dies bedingt, daB die Elemente der Mengen im folgenden gar nicht benutzet werden; immer nur bilden die an sich möglichen Beriehungen zwischen den Ganzen und ihren Teilen den Gegenstand der Untersuchung. Deren anf axiomatischer Grundlage rahende, umfassende Exōtterung bildet den eigentlichen Inhalt der Arbeit. Ieh habe aber doch die gewohnten Mengenbezeichnungen beibehalten. Fur die Ellemente der Mengese wird exst am SchluB eine anf 
den Begriff der Teilmenge sich stützende Einführungsmöglichkeit geżeigt. Sie erscheinen als solche Teilmengen, die selbst nicht weiter in Teilmengen zerlegbar sind (gleichsam als die Atome).

Bine zweite Eigenart der Untersuchung betrifft die logischen Notwendigkeiten, die der axiomatische Aufban dieses besondern Gebietes verlangt. Außer den selbstrerständlichen axciomatischen Festsetzungen über die Regeln, nach denen man mit den Begriffen der Äquivalenz, der Teilmengen usw. zu operieren hat, treten auch noch Annahmen auf, die man wohl nicht erwarten mag. Bei ihrer Binführang handelt es sich aber und darin besteht die genannte Eigenart - weniger um spezifisch mathematische Notwendigkeiten, als vielmehr um rein logische; also um Festsetzungen, die deshalb nötig sind, weil man ohne sie - um welches wissenschaftliche Gebiet es sich handeln mag - aus den. in Frage stehenden Voraussetzungen Schlüsse viberhaupt nicht ableiten kann. Ein allgemeiner Grundsatz der Logik lautet: E mere negativis nihil sequitur; $d . h$. aus lanter negativen Prämissen kann eine Folgerung nicht gezogen werden. Aus den Sätzen

kein $\mathfrak{A}$ ist ein $\mathfrak{B}$, kein $\mathfrak{B}$ ist ein $\mathfrak{C}$

läßt sich in der Tat eine Beziehung zwischen $\mathfrak{U}$ und $\mathbb{C}$ nicht entnehmen; und ebenso wenig gestatten die Sātze

kein $\mathfrak{B}$ ist ein $\mathfrak{X}$, kein $\mathfrak{C}$ ist ein $\mathfrak{X}$

eine Beziehung zwischen $\mathfrak{B}$ and $\left(\mathfrak{C}^{3}\right)$. Gerade solche Prämissen sind es aber, die uns bei den mengentheoretischen Problemen mehrfach begegnen, und deshalb der Finführung einer zwischen $\mathfrak{X}$ und $\mathbb{C}$ oder zwischen $\mathfrak{B}$ und $(5$ vorhandenen Beziehung den Stempel der axiomatischen Notwendigkeit anfdrücken.

\section{$\$ 1$. \\ Die Äquivalonz.}

Die mathematischen Objekte, von denen im folgenden die Rede sein wird, heifen Mengen (Teilmengen, Verbindungsmengen). Alle sollen den-

2) Aus den Vordersätzen

$\mathfrak{A}$ ist nicht $\mathscr{B}, \mathbb{Z}$ ist nicht $\mathbb{E}$

kann freilich in gewissen Fallen doch eine positive Folgerang gezogen werden und

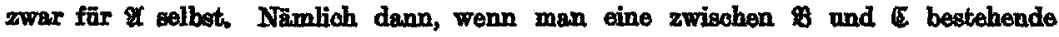
positive Bexiehung kennt. Aus den Sätzen:

Das Dreieck $D$ ist nicht spitzwinklig; und

Das Dreieck $\$$ ist micht stumpfwinklig

folgt, daB D rechtwinklig ist. Hier liegen nämlich nur acheinbar ausschließlich nogative Prämissen vor; zo ihnen kommat als positive der Satz: Jedes Dreieok ist entweder spitewinklig oder atumpfwinklig oder rechtwintlig. Vgl. auch die Anmerk, anf S. 182. 
selben Äquivalenzbeziehnngen gehorchen, die wir als Axiome der Aquivalens ( $\sim$ ) einführen. Sie lauten: Sind $M, N, P$ verschiedene Mengen, so gilt

$$
\begin{aligned}
& \text { I. Aus } M \sim N \text { folgt } N \sim M . \\
& \text { II. Aus } M \sim N \text { und } N \sim P \text { folgt } M \sim P .
\end{aligned}
$$

Der Aquivalenzbegriff hat also sowohl kommutativen, wie auch assoziativen Charakter.

Aus diesen Axiomen folgt:

1. Aus $M \sim N$ und $N$ nicht $\sim P$ folgt $M$ nicht $\sim P$. Denn wäre $M \sim P$, so würde daraus in Verbindung mit $N \sim M$ gemäß $I_{\text {s weiter }}$ $N \sim P$ folgen, im Gegensatz zur Voraussetzung.

Die Axiome I und II zeigen, daß sie die Ausdehnung auf den Fall zulassen, daß $M$ und $N$ dieselbe Menge bedeuten. Wir fïgen also als weiteres Axiom hinzu

$$
\text { III. Es ist } M \sim M \text {. }
$$

\section{$\S 2$.}

\section{Teilmengen and Verbindungsmengen.}

Ist $M^{\prime}$ Teilmenge von $M$, so soll dies durch

$$
M^{\prime} t M
$$

bezeichnet werden. Wir nehmen durchweg an, daB $M^{\prime}$ von $M$ verschieden ist, und nennen insofern $\boldsymbol{M}^{\prime}$ auch echte oder eigentliche Teilmenge von $\boldsymbol{M}$.

Für die Teilmengen sollen folgende Axiome gelten (Axiome der Teilmengen):

I. Aus $M^{\prime} t M$ und $M^{\prime \prime} t M^{\prime}$ folgt $M^{\prime \prime} t M$.

II. Jede Teilmenge $M^{\prime}$ von $M$ bestimmt eindentig eine zweite Teilmenge $M_{1}$ von $M$, die ihre Komplementärmenge bezïglich $M$ heißt.

III. Die Komplementärmenge von $M_{1}$ ist wiederum $M^{\prime}$.

Wir dürfen daher folgende Bezeichnungen einführen. Wir schreiben $M_{1} k M^{\prime}$ resp. $M^{\prime} k M_{1}$ und setzen demgemäB (III) in die Form

III'. Aus $M_{1} k M^{\prime}$ folgt $M^{\prime} \neq M_{1}$.

Für die Beziehung von $M_{1}$ und $M^{\prime}$ zur Menge $M$ selbst schreiben wir

$$
\boldsymbol{M}=\left(\boldsymbol{M}_{1}, \boldsymbol{M}^{\prime}\right)=\left(\boldsymbol{M}^{\prime}, \boldsymbol{M}_{1}\right)
$$

und sagen, daß $M$ in die Teilmengen $M^{\prime}$ und $M_{1}$ zerfältt. Zusammenfassend können wir also sagen:

Aus $M^{\prime} t M$ folgt $M_{2} t M, M_{1} k M^{\prime}, M^{\prime} k M_{1}, M=\left(M^{\prime}, M_{1}\right)$.

Seien num $M$ und $N$ zwei Mengen, so können beziiglich ihrer Teilmengen awei Fälle eintreten. Entweder gibt es für $M$ und $N$ identische, 
Teilmengen, oder es gibt keine solchen Teilmengen. In diesem Fall nennen wir die Mengen fremd zueinander, oder kurz fremd, und schreiben

$$
M f N \text { resp. } N f M \text {. }
$$

Für fremde Mengen gilt der Satz:

1. Sind $M$ und $N$ fremde Mengen, so ist anch jede Teilmenge von $M$ zu jeder Teilmenge von $N$ fremd; $d . h$.

Aus $M f N, M^{\prime} t M, N^{\prime} t N$ folgt $M^{\prime} f N^{\prime}$.

Wären nämlich die Teilmengen $\boldsymbol{M}^{\prime}$ und $N^{\prime}$ nicht fremd, und ist $\boldsymbol{P}$ eine in beiden enthaltene Teilmenge, so hätte man

$$
P t M^{\prime}, M^{\prime} t M \text { and } P_{t} N^{\prime}, N^{\prime} t \mathbb{N}^{\prime} \text {. }
$$

and daher gemäß I auch

$$
P \in \mathbb{M} \text { und } P t N \text {, }
$$

im Widerspruch mit der Voraussetzung.

1a. Der Satz gilt auch so, daB $M^{\prime}$ zu $N$ selbst, und ebenso $N^{\prime}$ zu $M$ fremd ist, Der Beweis ist derselbe.

Wir stellen weiter folgende Axiome auf:

IV. Die beiden Komplementärmengen $\boldsymbol{M}^{\prime}$ und $\boldsymbol{M}_{1}$ einer Menge $\boldsymbol{M}$ sind fremde Mengen; d. $\mathrm{h}$.

Aus $\boldsymbol{M}_{1} k \boldsymbol{M}^{\prime}$ folgt $\boldsymbol{M}_{1} f \boldsymbol{M}^{\prime}$.

Diese Beziehung soll aber auch umgekehrt gelten; zu diesem Zweck fuihren wir folgendes weitere Axiom ein (Axiom der Verbindungsmengen).

V. Zuei fremde Mengen $N$ und $P$ bestimmen eine und nur eine $M$ enge $\boldsymbol{M}$, deren Komplementärmengen sie sind; $\mathrm{d} . \mathrm{h}$.

Aus $N$ fP folgt $N t M, P t M$ und $N k P$.

Die Axiome IV und V lassen sich also auch so auffassen, dab die Beziehungen $N k P$ and $N f P$ gleichwertig sind. Wir nennen die Menge $(N, P)$ die Verbindungsmenge von $N$ und $P$. Es folgt noch

2. Die Mengen $N$ und $P$ sind von ihrer Verbindungsmenge $M=(N, P)$ verschieden.

Denn da sie nach $V$ Komplementärmengen von $M$ sind, so ist jede eine echte Teilmenge von $M$.

Die Menge $(N, P)$ hat außer $N$ und $P$ gemäB Axiom $I$ auch jede Teilmenge $N^{\prime}$ and $P^{\prime}$ an Teilmengen. Damit sind aber, wie wir durch ein weiteres Axiom festsetzen, nicht ihre sämtlichen Teilmengen erschōpft. Gemäl Satz 1 und 1 a ist auch $N^{\prime}$ zu $P^{\prime}$ fremd, ebenso $N^{\prime}$ zu $P$ und $N$ zu $P^{\prime}$; nach Axiom $V$ gibt es daher je eine Menge

$$
\left(N^{*}, P^{\prime}\right),\left(N, P^{\prime}\right) \text { and }\left(N^{\prime}, P\right) \text {. }
$$


Fï̈ sie setzen wir nun fest:

VI. Ist $M=(N, P)$, so sind auch die Mengen

$$
\left(N^{\prime}, P^{\prime}\right), \quad\left(N^{\prime}, P\right), \quad\left(N, P^{\prime}\right)
$$

Teilmengen von $M$; es ist aber auch jede von $N, N^{\prime} P, P^{\prime}$ verschiedene Teilmenge von dieser Frorm.

Wir folgern hieraus den Satz:

3. Ist $M=(N, P)$ and ist die Menge $Q$ fremd zu $N$ und fremd zu $P$, so ist sie anch fremd an $M$; d. h. aus $Q f N$ und $Q f P$ folgt $Q f(N, P)$.

Wäre nämlich die Menge $Q$ nicht fremd zn $M$, so gäbe es fuir sie und $\boldsymbol{M}$ eine identische Teilmenge; $d . h$. es gäbe eine Teilmenge $\boldsymbol{Q}^{\prime}$, die gemäß̊ VI eine der Formen.

$$
N, N^{\prime}, P, P^{\prime}, \quad\left(\mathcal{N}, P^{\prime}\right), \quad\left(N^{\prime}, P\right), \quad\left(N^{\prime}, P^{\prime}\right)
$$

haben müßte. Diese Teilmenge $Q^{\prime}$ hätte also jedenfalls $N$ oder $P$ oder eine Teilmenge von $N$ oder $P$ als Teilmenge; d. h. es gäbe eine Teilmenge $Q^{\prime \prime}$ von $Q^{\prime}$, die mit $N$ oder $P$ oder einer Teilmenge von $N$ oder $P$ identisch wäre. Nun ist aber nach $I Q^{\prime \prime}$ auch Teilmenge von $Q$ und damit exgibt sich ein Widerspruch gegen die Voraussetzung.

Der Satz 3 laßBt sich auch in die Form setzen:

3a. Ist die Menge $Q$ nicht fremd zux Menge $(\dot{N}, P)$, aber fremd zu $N$, so ist sie nicht fremd za $P$.

Will man den Begriff der Verbindungsmenge auf mehr als zwei Mengen ausdehnen, so hat man ein neues Axiom nötig. Fs ist jedoch für das Folgende nicht erforderlich, dies näher auszufiuhren.

\section{\$ 3.}

\section{Die Verknüptung der Mengen.}

Die verschiedenen Beziehungen, die zwischen zwei Mengen $\boldsymbol{M}$ und $\boldsymbol{N}$ Platz greifen können, sind aus der folgenden von Cantor angegebenen Aufzählung aller Möglichkeiten ersichtlich, die unsern Ausgangspunkt abgeben soll:

a) Es gibt ein $\boldsymbol{M}^{\prime} \sim \boldsymbol{N}$, und ein $\boldsymbol{N}^{\prime} \sim \boldsymbol{M}$.

b) Bs gibt kein $M_{1} \sim N$, aber ein $N^{\prime} \sim M$.

c) Es gibt ein $M^{\prime} \sim N$, aber. kein $N_{1} \sim M$.

d) Es gibt kein $M_{1} \sim N$, and kein $N_{1} \sim M^{4}$ ).

4) Die Anwendung oberer und unterer Indizes bei den Teilmengen im positiven ond negativen Fall soll im allgemeinen zar Erleichterang der Auffassung beibehalten wexden. 
Wir wollen diese vier Beziehungen durch

$$
M a N, M b N, M c N, \quad M d N
$$

darstellen. Man erkennt zunächst unmittelbar:

1. Die Bexiehungen a), b), c), d) schließen einander gegenseitig aus.

2. Die Beziehungen $M a N$ und $N a M$, ebenso $M d N$ und $N d M$ sind identisch. Die Beziehung $M b N$ ist identiseh mit $N \subset M$.

Wir erörtern sofort, welche dieser Beziehnngen sich auf den Fall ausdehnen lassen, daß $M$ und $N$ dieselbe Menge bedeuten. Es findet sich

2a. Die Beziehungen $M b M$ und $M c M$ sind widerspruchsvoll. Sie fordern nämlich das gleichzeitige Bestehen von

$$
M^{\prime} \sim M \text { und kein } M_{1} \sim M .
$$

Dagegen sind die Bexiehungen $M a M$ und $M d M$ widersprachsfrei. Ubrigens läßt sich dies auch als unmittelbare Folge von 1 und 2 auffassen.

Sei $P$ eine weitere Menge, so besteht zwischen $N$ und $P$ ebenfalls eine der Beziehungen

$$
N a P, N b P, N c P, N d P,
$$

und es entsteht die Frage, welche Folgerung sich für die Mengen $M$ und $P$ einstellt, wenn man eine Beziehung der Reihe (A) mit einer Beziehung der Reihe (B) kombiniert. Diese Aufgabe läßt sich ohne Einführung neuer Axiome nicht erledigen. Ein exstes, das den Begriff der Teilmenge mit dem der Äquivalenz verbindet, sei das folgende:

I. Aus den Relationen

lassen sich die Relationen

$$
M^{\prime} t M, M \sim N
$$

$$
N^{\prime} t N, N^{\prime} \sim M^{\prime}
$$

folgern; d. h. ist $M \sim N$, so bedingt eine jede Teilmenge $M^{\prime}$ von $M$ die Existenz einer Teilmenge $N^{\prime}$ von $N$, die zu $M^{\prime}$ äquivalent ist.

Vielleicht mag man erwarten, daß die Menge $N^{\prime}$ als diejenige wohlbestimmte Menge eingefiüht wird, die der Menge $\boldsymbol{M}^{\prime}$ gemäß der zivischen $M$ und $N$ bestehenden Aquivalenz entspricht. Aber dies ist für den hier vorgenommenen Aufbau - jedentalls an dieser Stelle - weder möglich noch nötig: Es genügt, die Existenz einer Menge $N^{\prime}$ zu forderm; welches diese Menge ist, daxf ganz offen bleiben. Hs hängt dies damit zusammen, daf die Äquivalenz $M \sim N$ in ihree besondern Figenart hier nicht in Frage kommt; nur die Relationen, die die Eigenart des Aquivalenzbegriffs kennzeichnen, and tür zwei Mengen und ihre Teilmengen bestehen, werden in Betracht gezogen. 
Binen Toil der oben gestellten Frage hat bekanntlich schon Cantor sellbst beantwortet; man zeigt leicht

3. Aus $M a N$ und $N a P$ folgt $M a P$.

4. Aus $M a N$ und $N b P$ folgt $M b P$.

5. Aus $M a N$ und $N e P$ folgt $M e P$.

6. Aus $M b N$ und $N b P$ folgt $M b P$.

7. Aus $M C N$ and $N e P$ folgt $M c P^{s}$ ).

Die Beweise sing natürlich ausschlieBlich auf die in a), b), c), d) enthaltenen Bexiehungen zu stützen. Fin Beispiel möge zeigen, wie sie sich führen lassen. Um aus den Relationen

$M b N$ und $N B P$ weiter $M B P P$

zu folgern, haben wir von

$$
\begin{aligned}
& \text { kein } M_{1} \sim N, \quad \text { ein } N^{\prime} \sim M, \\
& \text { kein } N_{2} \sim P, \quad \text { ein } P^{\prime} \sim N
\end{aligned}
$$

auscugehen ${ }^{5 a}$ ), und darans die Bexiehungen

$$
\text { kein } \cdot M_{1} \sim P, \text { ein } P^{\prime} \sim M
$$

abzaleiten. Wir beweisen zunächst den zweiten Teil. Wegen $P^{\prime} \sim N$ gibt es nach $I$ eine Teilmenge $P^{\prime \prime} \sim N^{\prime}$, und aus $N^{\prime} \sim M$ folgt nun $P^{\prime \prime} \sim M$. Die Richtigkeit der ersten Behauptung erweisen wir indirekt. Wäre nämlich ein $M^{\prime} \sim P$, so folgte gemäB I aus $N^{\prime} \sim M$ wiederum die Existenz einer Menge $N^{\prime \prime}$ von $N^{\prime}$, für die $N^{\prime \prime} \sim M^{\prime}$ sein müßte, und aus

$$
M^{\prime} \sim P, N^{\prime \prime} \sim M^{\prime} \text { weiter } N^{\prime \prime} \sim P \text {, }
$$

während kein $N_{1} \sim P$ sein kann.

Es bleibt noch übrig, das gleichzeitige Bestehen der Beziehungen

$$
M b N \text { und } N C P
$$

za untersuchen, sowie die Kombination von $M d N$ mit einer der Beziehangen

$$
N a P, N b P, N \subset P, N d P \text {. }
$$

Hier gilt zanächst, $d a B$ ans $M b N$ und $N C P$ eine bestimmte $B e$ ziehung zwisehen $M$ und $P$ nicht folgt; d. h.

8. Mit $M b N$ und $N c P$ ist jede der vier Beziehungen $M a P, M b P$, $M c P, M d P$ vertzäglich.

5) Diese Tatsachen entsprechen bekanntlich dem Umstand, daB wenn man den

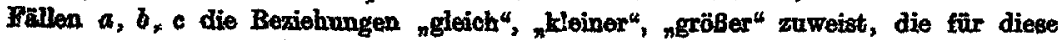
Bexiebnangen geltenden ascoziativen Gesetze erfäll sind (z. B. ans $\alpha=\beta$ und $\beta=\gamma$ folgt $\alpha=y$ usw.).

54) De klax, daB $N^{\prime} \sim M$ usw. picht die Bedeutang hat von "nur ein“ sondern ron mindestons ein ${ }^{\alpha}$. 
Der Beweis darf unterbleiben. Nur sei bemerkt, daß dies dem realen Tatbestand entspricht, dessen axiomatisahe Grundlegung hier in Frage steht ${ }^{6}$ ).

Wir gehen nun an dem Rest unseres Problems über und prüfen zunächst die Kombination von

$M d N$ und $N d P$.

Die Frage lautet auch hiex, ob die Bexiehangen ( $\alpha$ ) eine bestimmte Beziehung zwischen $M$ und $P$ bedingen und eventuell welche. Hier liegt der in der Finleitung genannte Fall vor, daß es sich um lauter negative Prämissen handelt. Diese Prämissen sind

$$
\begin{cases}\text { kein } M_{1} \sim N, & \text { kein. } N_{1} \sim M, \\ \text { kein } N_{1} \sim P, & \text { kein } P_{1} \sim N .\end{cases}
$$

Aus ihnen läßt sich auf direktem Wege über die Beziehung von $M$ zu $P$ nichts schließen. Teilweise gelingt es allerdings ouf indirektem Wege; in einzelnen Fallen kommt nämlich dadurch zu den obigen Prämissen eine neue Tatsache hinzu, die positiver Natur ist. Um die Untersuchung durchrufibihren, hat man nämlich zu prüfen, ob die Annahme einer der Beziehungen

$$
M a P, M b P, M c P, M d P
$$

auf Grund der bisherigen axiomatischen Festsetzungen einen Widerspruch mit dem gleichzeitigen Bestehen der Beziehungen ( $\alpha$ ) bedingt, und zwar kommen naturgemäB hier nur die Axiome von \$1, das obige Axiom \$ 3, I und der obige Satz 2 in Frage. Diese Prüfung haben wir ausführlich vorzunehmen ${ }^{7}$ ).

Zunächst sieht man leicht, daß die Beziehungen

$$
M b P \text { and ebenso } M \subset P^{\prime}
$$

als Folgen von $(\alpha)$ anszuschließen sind. Wegen Satz 2 kann man nämlich die Beriehungen $(\alpha)$ auch in die Form

$$
P d N \text { und } N d M
$$

setzen, und müBte daher als Folge von ( $\alpha$ ) ebenso auch

$$
P b M \text { oder } P c M
$$

erhalten. Aber $M b P$ und $P b M$, und ebenso $M C P$ und $P C M$ sind nach Satz 2 nicht identisch, womit die Behanptung erwiesen ist $\left.{ }^{8}\right)$.

O) Für Mäohtigkeiten würden die Relationen $m<n$ and $n>p$ bestehen; vie bedingen keine Größenbeziehung zwischen m and $\mathfrak{p}$.

7) In den Math. Ann. 72 (1912), S. 551, iat diese Untersuchung schon teilweise durchgeführt worden.

7) Die logische Eigenart des oben behandelten Problems entopricht also nicht ganz dem in dex Binleitmog genannten Tatbestand. Ds lautet nämlich genauer an; 
Es ist weiter 20 untersuchen, ob sich die Beziehung

\section{$\operatorname{MaP}$}

als Folge von ( $\alpha$ ) einstellen kann. Hiex ist ein Resultat, das dies unmöglich macht, nicht erhältilich. Die Bexiehung $M a P$ bedeutet nämlich

$$
\text { eins } M^{\prime} \sim P, \text { ein } P^{\prime} \sim M .
$$

Die Verbindung mit $(\alpha)$ liefert gemäß $\$ 1$ die weiteren Relationen

$$
\operatorname{kein} N_{1} \sim P^{\prime}, \quad \text { kein } N_{1} \sim M^{\prime} \text {. }
$$

Genauer bedentet dies: Es gibt eine Teilmenge $P^{\prime}$, der keine Teilmenge von $N$ äquivalent ist, und es gibt auch eine Teilmenge $M^{\prime}$, der keine Teilmenge von $N$ äquivalent ist. Dies stellt aber einen Widerspruch zu $\left(\boldsymbol{\alpha}^{\prime}\right)$ oder zu $\left(\boldsymbol{\gamma}^{\prime}\right)$ nicht dar.

Es soll noch eine zweite Prüfung vorgenommen werden; wir haben auch den assoziativen Charakter der Beziehungsregeln in Betracht zu ziehen. Ist MaP das Resultat von $(\alpha)$, so heißt dies, daß das gleichzeitige Bestehen von

$$
M d N, \quad N d P, \quad M a P
$$

nicht widerspruchsvoll sein darf. Nun sollen aber zwei von diesen Bedingungen stets eine dritte bedingen, und darans folgt, dab aus

und aus

$$
M a P \text { und } P d N \text { wieder } M d X
$$

\section{$N d M$ und $M a P$ wieder $N d P$}

folgen muß. Es ist nun die Frage, ob diese Regeln einen widerspruchslosen Charakter haben. Dies ist in der Tat der Fall. Man sieht es am einfachsten daraus, da8 man die assoziativen Gesetze, die die Beziehungen

Wolche von vier möglichen Bexiehungen wixd duroh die dem Problem eigentïmlichen nur negativen Prämissen ansgeschlossen? Bei der Annahme, MbN oder McN seien die Folgen dieser negativen Prämissen, wird von selbst eine nene Tatseche eingeführt; die Symmetrie der Bexiehungen $M d N$ und NdP bezüglich $M$ and $P$ steht nämlich im Gegensatz zo der Unsymmetrie der Folgerungen $M b P$ oder $M c P$ für $M$ und $P$. Ond daher exgab gich oben ein Rosultat. Die Annohme, MaP oder MdP seien die Folgen der negativen Prämissen, liefert dagegen eine solohe neue Tatsache nicht; es ergibt sich daher, wie das obige weiter zeigt, ein Resultat nicht.

Allgameiner gesprochen: Wenn die Prämiscen: $\mathfrak{A}$ ist nicht $\mathscr{B}$ und $\mathfrak{B}$ ist nicht $\mathbb{C}$

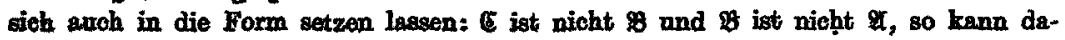

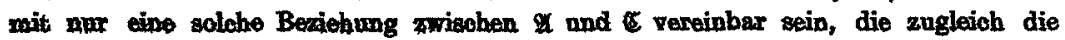

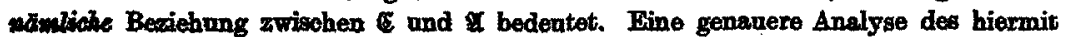
mehrfach besprochenen logüechan Problems von seiten dex Logiker wäre sehr erwünscht. Das lotzte Wort wall mil dew vorstehenden nicht gesprochen sein. 
(a) und (d) miteinander verbinden, wenn man noch Satz 3 beschtet, in die einfache Form

$$
(a a)=(d d)=a, \quad(a d)=(d a)=d
$$

setzen kann; sie sind das genaue Analogon zu den Vorzeichenregeln

$$
(+)(+)=(-)(-)=+; \quad(+)(-)=(-)(+)=-,
$$

deren assoziativer Gesamtcharakter feststeht.

Wir haben endlich noch die Beziehung

\section{$M d P$}

als mögliche Folge der Beziehungen $(\alpha)$ zu erörtern. Sie bedeutet kein $M_{1} \sim P, \quad$ kein $P_{1} \sim M$.

Hier zeigt sich zunächst, daB sich aus ihr und den Relationen $\left(\alpha^{\prime}\right)$ weitere direkte Folgerungen überhaupt nicht entnehmen lassen, da sie jetzt samt und sonders negativer Natur sind. Wir prüfen auch hier noch den assoziativen Gesamtcharakter. Ist $M d P$ das Resultat von $M d N$ and $N d P$, so bedingt es jetzt, daB aus

$M d P$ und $P d N$ wieder $M d N$

nnd ans

$M d M$ und $M d P$ wieder $M d P$

folgt; hier aber iṣt der widerspruchsfreie Charakter evident. Also folgt:

9. Mit den Beziehungen $M d N$ und NdP kann sowohl die Beziehung $M a P$, wie $M d P$ zugleich bestehen:

Keine der beiden Annahmen $\gamma$ und $\delta$ führt also auf einen Widerspruch mit den in $\left(\alpha^{\prime}\right)$ enthaltenen Prämissen; wir können daher auf diesem Wege nicht zu einem Resultat über die vorliegende Frage gelangen. Man muß daher in der Tat die Folgerung, die sich aus $M d N$ und $N d P$ ergeben soll, axiomatisch einfühtren; naturgemäß so, wie es durch den realen Tatbestand der Mengenlehre gefordert wird. Ihn aufaubauen ist ja einer der $Z_{\text {wecke dieser Darstellung }}{ }^{2}$ ). Wir setzen daher fest (Axiom der Verknüpfung)

\section{Aus $-M d N$ und $N d P$ folgt $M d P$.}

Dieses Axiom hat einen doppelten Charakter, einen rein logischen, und einen mathematischen. 'Es enthält die logische Forderung, dab aus zwei bestimmten Prämissen der Reihen (A) und (B) - wenn sie nicht etwa

9) Das obige Axiom entspricht in Rahmen der Cantorschen Theorie inhaltiioh der Forderung der Vergleiohbarkeit. Der Text zoigt, daB diesar Forderrong eine gewisse rein logische Bedeutung inne wohnt. Man kann natürlich eine Mengenlehre auch so aufrubanen suchen, daB man die Unvergleichbarkeit als möglich zulaßt. Davon ist hier abgesehen worden; vgl. übrigens auch $\$ 7$. 
einander widersprechen - stets ein und nur ein Schluß gefolgert wenden soll; auBerdem die mathematische, daB es gerade der in II enthaltene sein soll. Ans ihr erhalten wir nun leicht die Antwort anf die noch ausstehenden Verknüpfungen fïr die Beziehungen (A) und (B). Zunächst beweist man

10. Aus $M b N$ and $N d P$ folgt $M b P$.

10a. Aus $M C N$ und $N d P$ folgt $M c P$.

Für den Beweis von (10) haben wir auszugehen von

$$
\begin{aligned}
& \text { kein } M_{1} \sim N, \quad \text { ein } N^{\prime} \sim M, \\
& \text { kein } N_{1} \sim P, \quad \text { kein } P_{1} \sim N,
\end{aligned}
$$

und daraus die Beziehung $M b P$, also

$$
\text { kein } M_{1} \sim P, \text { ein } P^{\prime} \sim M
$$

abzuleiten. Wir folgern zanächst, dab eine Bexiehung

$$
M^{\prime \prime} \sim P
$$

unmöglich ist. Aus $N \sim M$ würde nämlich auf Grund dieser Annahme die Existenz einer Teilmenge $N^{\prime \prime}$ folgen, für die

$$
N^{\prime \prime} \sim \boldsymbol{M}^{\prime \prime} \sim P
$$

wäre, im Widersprach zu kein $N_{1} \sim P$. Damit ist die Bexiehung kein $M_{1} \sim P$ erwiesen. Es ist jetzt noch zu zeigen, daB es ein $P^{\prime} \sim M$ gibt. Wäre dies nicht der Frall, so bestände auf Grund des vorstehenden jetżt die Beziehung

$$
\text { kein } M_{1} \sim P, \quad \text { kein } P_{1} \sim M,
$$

also die Relation $M d P$, und zusammen mit der voransgesetzten Beziehung $P d N$ folgte gemäB Axiom II die Beziehung $M d N$, im Widerspruch zu MbN. Damit ist der Beweis wieder geliefert. Ebenso wird der Beweis für $M \subset N$ und $N d P$ geführt, was einer ausführlichen Darstellnng nicht. bedarf.

Wir haben schließlich noch die Kombination von

$$
M a N \text { und } N d P
$$

zu exörtern. Wir folgern zunächst, daß diese beiden Relationen an sich nur die Folge

$$
M d P
$$

gestatten. Wir haben asszugeben von

$$
\begin{aligned}
M^{\prime} & \sim N, \quad N^{\prime} \sim M, \\
\text { kein } N_{1} & \sim P, \quad \text { kein } P_{1} \sim N,
\end{aligned}
$$


und zeigen munächst, daß hiermit nur

$$
\text { kein } M_{1} \sim P, \quad \text { kein } P_{1} \sim M,
$$

verträglich sind. Gäbe es nämlich eine Menge $M^{\prime \prime} \sim P$, so folgerte man wie oben eine Relation

$$
N^{\prime \prime} \sim M^{\prime \prime} \sim P
$$

im Widerspruch mit der Voraussetzung: kein $N_{1} \sim P$; ebenso folgt die Unmöglichkeit einer Beziehung $P^{\prime \prime} \sim M$. Es kann also an sich nur die Relation

\section{$M d P$}

bestehen. Wiederum ist noch der assoziative Charakter des Resultats zu prüfen. Diese Prüfung führt hier auf einen Widerspruch. Ans MdP und $N d P$ würde nämlich gemäB dem Axiom II $M d N$ folgen, im Widerspruch mit der Annahme MaN. Das gleichzeitige Bestehen von MaN and $N d P$ führt also auf einen Widerspruch; $d . h$. stehen.

11. Die Bexiehungen MaN und NdP können nicht zugleich be-

Dagegen sei ausdrücklich festgestellt, daB dis Sãtze 10 und 10a einen solchen Widersprach nioht herbeiführen. Denn gemäB Satz 2 ist MbP mit $P c M$ identisch, und die beiden Beziehungen

$$
P c M \text { and } M b N
$$

sind, wie wir oben unter 8. enwähnten, mit jeder der vier an sich möglichen Beziehungen zwischen $N$ und $P$ verträglich.

Damit ist unsere Untersuchung abgeschlossen; sie zeigt zugleich die Widerspruchslosigkeit des Axioms II. Wir ziehen ans ihm zunächst noch eine Folgerang, nämlich die, daß der Satz 11 auch in der Weise gilt, daB er das gleichzeitige Bestehen von

$$
M a M \text { und } M d N \text {, sowie von } M a N \text { und } N d N
$$

ausschließt. Aus $M a M$ folgt $M^{\prime} \sim M$ und hieraus gemäB $\$ 3, I$

$$
M^{\prime \prime} \sim M^{\prime} \sim M
$$

und daher besteht auch die Relation

$$
M^{\prime} a M \text {; }
$$

diese kann aber nach Satz 11 nicht mit MdN zugleich bestehen.

Weiter folgt aus MaN zunächst

$$
\boldsymbol{L}^{\prime} \sim \boldsymbol{N}, \quad \boldsymbol{N}^{\prime} \sim \boldsymbol{M},
$$

also auch $N^{\prime \prime} \sim M^{\prime} \sim N$, während dagegen $N d N$ besagt, daB kein $N_{1} \sim N$ ist. Also 
11 a. Die Beziehungen $M a M$ und $M d N$, ebenso $M a N$ und $N d N$ schliepen einander aus.

Es ergibt sich damit das folgende Schlußresultat. Mit den Beziehrungen $M d N$ und $N d P$

erscheint sowohl die Folgerung $M a P$ wie auch die Folgerung MdP verträglich. Wird die Relation $M d P$ axiomatisch als Folgerung eingeführt, so bedingt dies, daß die Beziehungen $M a N$ und $N d P$ nicht zugleich bestehen können; würde man dagegen die Beziehung MaP axiomatisch als Folgerung einführen, so ergibt sich ein derartiges Resultat nicht. Trotzdem erfordert der Aufbau der Mengenlebre die Einführung der Folgerung $M d P$. Anf die Deutungsmöglichkeit der axiomatischen Ànnahmo MaP komme ich in $\$ 7$ aurück.

Für die Beziehungen $(a),(b),(c),(d)$ gelten noch die folgenden besonderen Sätze:

12. Aus den Relationen

und

$$
M a N, M b N, M c N, M d N
$$

folgt auch

und

$$
M \sim \mathfrak{M}, \quad N \sim \mathfrak{R}
$$

$\mathfrak{M a N}, \dot{M} b N, \operatorname{Mc} N, \operatorname{Mt} d N$

$M a \Re, \quad M b \Re, \quad M c \Re, \quad M d \Re$.

Für den Beweis mag ein Beispiel genügen. Werde von

$$
M b N \text { and } M \sim M
$$

ausgegangen, so heibt dies

$$
N^{\prime} \sim M_{2} \text { jedes } M_{1} \text { nicht } \sim N .
$$

Wir erhalten daher, falls $\mathfrak{M}_{1} \sim M_{1}$ ist, gemäB $\S 1$ sofort

$$
N^{\prime} \sim \mathfrak{M}, \quad \text { jedes } \mathfrak{M}_{1} \text { nicht } \sim N,
$$

womit die Behauptung ezwiesen ist.

13. Aus $M^{\prime} t M$ folgt $M^{\prime} a M$ oder $M^{\prime} b M ; d . h$. für jede $T$ eil menge $M^{\prime}$ gilt entweder $M^{\prime} a M$ oder $M^{\prime} b M$.

Es gibt nämlich eine Teilmenge von $M$, die äquivalent $\boldsymbol{M}^{\prime}$ ist, nämlich $\mathcal{M}^{\prime}$ selbst, und daher ist die Beziehung $(c)$ und $(d)$ ausgeschlossen.

14.. Aus $M^{\prime} t M$ und $M b N$ folgt $M^{\prime} b N$; d.h besteht die Beziehning $M b N$, so besteht für jede Teilmenge $M^{\prime}$ von $M$ die Beziehung $M^{\prime} b N$.

Man hat nämlich gemäB 13. und nach Voraussetzang

$$
M^{\prime} a M \text { oder } M^{\prime} b M \text { and } M b N \text {, }
$$

und damit gemäB Satz 4 und 6 die Behauptung. 
15. Aus $M^{\prime} t M, M^{\prime \prime} t M^{\prime}, M^{\prime \prime} b M^{\prime}$ folgt $M^{\prime \prime} b M$; d. h. sind $M^{\prime}$ ind $M^{\prime \prime}$ Teilmengen von $M$, für die die Beziehung $M^{\prime \prime} b M^{\prime}$ gilt, so ist auch $M^{\prime \prime} b M^{10}$ ).

Man hat nämlich wieder augleich (nach 13.)

$$
M^{\prime \prime} b M^{\prime} \text { und } M^{\prime} a M \text { oder } M^{\prime} b M
$$

und folgert daraus wie eben $M " b M$.

$$
\S 4 .
$$

Endliche und unendliche Mengen.

Nach $\S 3$, Satz 1 und 2 sind $M a M$ und $M d M$ die beiden einzigen der Beziehungen $(a),(b),(c),(d)$, die eine Menge zu sich selbst haben kann; wir definieren nun: 1. Eine Menge heibt unendlich, wenn die Beziehung $M a M$ besteht; sie heißt endlich, wenn $M d M$ gilt. Man hat also im ersten oder zweiten Fall

$$
\text { ein } M^{\prime} \sim M ; \text { kein } M_{1} \sim M,
$$

und damit die Dedekindsche Begriffsbestimmung.

Wir folgern zunächst:

2. Aus $M a M$ oder $M d M$ and $M \sim \mathfrak{M}$ folgt $\mathfrak{M} a \mathfrak{M}$ und $\mathfrak{M} d \mathfrak{M}$. Dies ist eine unmittelbare Folge von $\$ 3,12$.

Für endliche und unendliche Mengen bestehen gewisse Sondersätze; diese sollen jetzt abgeleitet werden. Das Haupttheorem lautet:

3. Für unendliche Mengen können nur die Bexiehungen $(a),(b),(c)$ bestehen; für endliche Mengen nur (b), (c), (d).

Der Beweis ergibt sich unmittelbar aus den in $\$ 3$ abgeleiteten Resultaten.

Sind nämlich $M$ und $N$ unendliche Mengen, und würde die Beziehung $M d N$ bestehen, so hätte man

$$
M a M \text { and } M d N \text {, }
$$

und dies verstößt gegen Satz 11 a von $\$ 3$.

Ebenso, wenn $M$ und $N$ endliche Mengen sind, so hätte man, falls sie die Beziehung MaN gestatten,

$$
N a M \text { und } M d M \text {, }
$$

und anch dies verstöbt gegen Satz 11 a von $\$ 3$.

Damit ist der Satz 3 bewiesen. Er gibt zugleich den inneren Grund für die im Satz 11 von $\$ 3$ enthaltene Unvereinbarkeit von $M a N$ and

10) Dieser Satz berïht aich inhaltlich mit dem Satz 25 in Zermelos Grundlagen (Math. Ann. 65, S. 271). 
$\boldsymbol{N} \boldsymbol{M}$ * Denn unserem Satz 3 gemäß besagt $M a N$, daB $M$ und $N$ unendliche Mengen sind, und $N d P$, daß $N$ und $P$ endliche Mengen sind. Beides schließt sich aber aus.

4. Für jede Teilmenge einer endlichen Menge besteht die Bexiehung $M^{\prime} b M$; d. b. aus $M d M$ und $M^{\prime} t M$ folgt $M^{\prime} b M$.

Gemäß Satz 13 von $\$ 3$ gilt nämlich für jede Menge $M$ und eine Teilmenge $\boldsymbol{M}^{\prime}$ von ihr

\section{$\boldsymbol{M}^{\prime} \boldsymbol{a} \boldsymbol{M}$ oder $\boldsymbol{M}^{\prime} b \boldsymbol{M}$.}

Hierza kommt, da $\boldsymbol{M}$ eine endliche Menge ist, $\boldsymbol{M} d \boldsymbol{M}$. Diese Beziehung kann aber nach Satz 11 von $\$ 3$ mit $M^{\prime} a M$ nicht zugleich bestehen; also muß es $\boldsymbol{M}^{\prime} b \boldsymbol{M A}$ sein.

Die weiteren noch abzuleitenden Sätze machen die Einführung eines neuen Axioms nötig, und zwar eines Axioms ïber die Aquivalenz von Verbindungsmengen. Hs lautet:

I. Aus $M=(N, P), N \sim \Re, P \sim \Re, \Re f \Re$ folgt $(N, P) \sim(\mathfrak{N}, \mathfrak{B})$; d. h. verden in der Verbindungamenge $(N, P)$ die Mengen $N$ und $P$ durch die su ihnen äquivalenten, zueinander fremden $M$ engen $\mathfrak{N}$ und $\mathfrak{P}$. ersetat, so ist die neue Menge der ursprüinglichen äguivalent.

Das Axiom gilt gemäB §1, III auch für den Fall, daß nur eine Yenge durch eine äquivalente ersetat wird, $d . h$.

5. Aus $M=(N, P), N \sim \mathfrak{R}, \mathfrak{R} f P$ folgt $\left.(N, P) \sim(\Re, P)^{12}\right)$.

Wir beweisen nun der Reihe nach folgende Sätze:

6. Jede Teilmenge einer endlichen Menge ist selbst eine endliche Menge; d. h. ans $M d M, M^{\prime} t M$ folgt $M^{\prime} d M^{\prime}$.

Wầre nämlich $\boldsymbol{M}^{\prime}$ eine unendliche Mengè, so müßte eine Beziehung

bestehen. Setzt man nun

$$
\boldsymbol{M}^{\prime \prime} \sim \boldsymbol{M}^{\prime}
$$

so ist gemäB $\$ 2$ VI anch

$$
M=\left(M^{\prime}, M_{1}\right)
$$

$$
M^{\prime \prime \prime} \sim\left(\boldsymbol{M}^{\prime \prime}, M_{1}\right)
$$

eine Teilmenge $\nabla$ on $M$ und aus Satz 5 folgte

$$
\boldsymbol{M}^{\prime \prime \prime} \sim \boldsymbol{M}
$$

was einen Widersprach gegen $M d M$ darstellt.

7. Ist $M$ eine exdliche, $N$ eine unendliche Menge, so kann nur die Besiehung $M b N$ bestehen $\left.{ }^{19}\right) ;$ d. h ans $M d M$ und $N a N$ folgt $M b N$.

m) Is liegt nohe, Satz 5 als Axion hinzustellen, und das Axiom als Folge. Der Bewreis hätte aber dit sachlich öberfitasige Annahme $\$ f P$ nötig.

- Auf dien Sakz wurdo ich sohon vor langerer Zeit von Herm H. Hahn anfmeriseam gemacht. 
Der Beweis wird so getährt, daß die Unvereinbarkeit der Voraussetzangen mit $M a N, M c N, M d N$ gezeigt wird.

Würde zunächst die Beziehung $M a N$ bestehen, so hätte man $M^{\prime} \sim N$; und demgemäß exhielte man aus der Annahme MaN nach § 3 Satz 12 weiter auch

$$
M a M^{\prime} \text { resp. } M^{\prime} a M \text {, }
$$

was aber, da $M$ endliche Menge ist, gegen Satz 4 verstöBt.

Wäre eweitens $M c N$ in Kraft, so folgte daraus $M^{\prime} \sim N$, und nun hiereus und aus $\mathrm{NaN}$ weiter

$$
M^{\prime} a M^{\prime} \text {, }
$$

was wiederum einen Widerspruch zum Satz 6 darstellt.

Endlich ist auch die Beziehung $M d N$ unmöglich. Denn aus $N a N$ folgt zunächst

$$
N^{\prime} \sim N ;
$$

hieraus und aus $N a N$ und der angenommenen Relation $M d N$ folgte dann weiter

$$
N a N^{\prime} \text { and } M d N^{\prime} \text { resp. } N^{\prime} d M \text {. }
$$

Die Bexiehungen $N a N^{\prime}$ und $N^{\prime} d M$ sind aber gemäB $\$ 3$ Satz 11 nicht zugleich möglich. Also gilt in der Tat die Beziehung MbN.

8. Ist $M$ eine unendliche Menge, so ist auch die Verbindungsmenge $(M, N)$ eine unendliche Menge.

Der Beweis ist eine unmittelbare Folge des Axioms I. Denn

$$
\text { aus } \boldsymbol{M}^{\prime} \sim \boldsymbol{M} \text { folgt }(\boldsymbol{M}, \boldsymbol{N}) \sim\left(\boldsymbol{M}^{\prime}, N\right),
$$

und damit ist der Satz, da $\left(M^{\prime}, N\right)$ Teilmenge von $(M, N)$ ist, bewiesen.

9. Fine Menge ist unendlich, wenn sie eine unendliche Teilmenge hat.

Ist nämlich $\boldsymbol{M}^{\prime}$ diese Teilmenge, so ist

$$
M=\left(M^{\prime}, M_{1}\right)
$$

und daher gemäß Satz 8 auch $M$ eine unendliche Menge.

Man kann diesen Satz anch noch so formulieren:

$9^{\prime}$. Eine Menge ist endlich, wenn jede ihrer Teilmengen endlich ist.

10. Ist $M$ eine endliche $M$ enge, so ist stets $M b(M, N) ; d . h$ aus $M d M$ folgt $M b(M, N)$.

Es ist nämlich $M$ Teilmenge von $(M, N)$. Ist nun $(M, N)$ endlich, so folgt der Satz aus 6 , ist aber $(M, N)$ unendlich, so folgt er' aus 7.

Zar Ableitung weiterer Sătze bedürfen wir neuer Axiome. Das Axiom I besagt, dal die Verbindungsmengen äquivalenter Mengen selbst aquivalent sind; wir haben jetat noch zwei Axiome nötig, die die Nichtagrivalenz der Verbindungomengen nicht äquivalenter Mengen betreffen. 
II. Sind $M$ und $N$ fremde Mengen, ist $M_{1}$ Teilmenge ron $M$ und $N_{1}$ Teilmenge von $N$, und ist $M_{1}$ nicht $\sim M, N_{1}$ nicht $\sim N$, so folgt daraus die Beziehung $\left(M_{1}, N_{1}\right)$ nicht $\sim(M, N) ; \mathrm{d}$. h. aus $M \nmid N, M_{1} t N$, $N_{1} \neq N, M_{1}$ nicht $\sim M, N_{1}$ nicht $\sim N$ folgt $\left(M_{1}, N_{1}\right)$ nicht $\sim(M, N)$.

Dieses Axiom soll für alle Mengen gelten. Für endliche Mengen reicht aber noch nicht aus, und werde durch das folgende ersetzt und ergänzt:

III. Sind $M$ und $N$ fremde und zugleich endliche Mengen, und ist $M_{1}$ Teilmenge von $M$, so soll stets $\left(M_{2}, N\right)$ nicht $\sim(M, N)$ sein, d. $h$. aus $M f N, M d M, N d N, M_{1} t M$ folgt $\left(M_{1}, N\right)$ nicht $\sim(M, N)$.

Für unendliche Mengen braucht dieses Axiom bekanntlich nicht erfüllt zu sein.

Auch die Voraussetzungen dieser Axiome besitzen durchaus den in der Einleitung genannten logischen Sondercharakter; sie sind sämtlich negativer Natur, soweit es sich um die hier allein in Frage stehenden Iquivalenzbeziehungen handelt. Man könnte freilich annehmen, daB in diesem Fall ein indirektes Beweisverfahren zum Ziele führen werde; die Annahme

$$
\left(M_{1}, N_{1}\right) \sim(M, N) \text { resp. }\left(M_{1}, N\right) \sim(M, N)
$$

ist ja von positivem Charakter. Aber diese Vermutung trügt. Die Åquivalenz von Verbindungsmengen ist nämlich keineswegs nur so möglich, daB $M_{1} \sim M$ und $N_{1} \sim N$ ist, sondern auch auf andere Weise; und daher kann aus der angenommenen Äquivalenzbeziehung ein Widerspruch mit den Voraussetzungen

$$
M_{1} \text { nicht } \sim M, \quad N_{1} \text { nicht } \sim N
$$

nicht abgeleitet werden.

Die negative Fassung unserex Axiome stellt uns zunächst vor die Aufgabe, die bestimmte Bexiehung $(a),(b),(c),(d)$ aufzufinden, die zwisehen $(M, N)$ and den Mengen $\left(M_{1}, N_{1}\right)$ und $\left(M_{1}, N\right)$ besteht. Für das Axiom II kann es erst im nächsten Paragraphen geschehen; für das Axiom III soll es hier folgen.

Da $\left(M_{1}, N\right)$ Teilmenge von $(M, N)$ ist, so kann nach Satz 13 von $\$ 3$ nur die Beziehung $(a)$ oder $(b)$ realisiert sein. Aber der Fall $(a)$, d. h.

$$
\left(M_{2}, N\right) a(M, N)
$$

ist unmöglich. Jede Teilmenge von $\left(M_{2}, N\right)$ hat nämlich nach $\$ 2, \mathrm{VI}$ eine der Formen

$$
M_{1}, M_{4}, N, N_{1},\left(M_{3}, N\right),\left(M_{1}, N_{1}\right),\left(M_{2}, N_{1}\right),
$$

wo $\boldsymbol{H}_{9}$ eine Teilmenge von $M_{1}$ ist. Keine von ihnen kann aber zu 
$(\boldsymbol{M}, \boldsymbol{N})$ äquivalent sein. Da nämlich $\boldsymbol{M}$ und $\boldsymbol{N}$ endliche Mengen sind, so hat man fuir sie gemäB 10. die Relationen

$$
M b(M, N) \text { und } N b(M, N) \text {. }
$$

GemäB Satz 4 hat man weiter

$$
M_{1} b M, \quad M_{2} b M, \quad N_{1} b N
$$

und damit folgt die Behauptung nach Satz 6 von $\$ 3$ bereits für $M_{1}, M_{2}, N, N_{1}$. Für die drei Verbindungsmengen folgt sie aus den Axiomen selbst; es ist ja, da $M$ und $N$ endliche Mengen sind,

$$
M_{1} \text { nicht } \sim M, \quad M_{2} \text { nicht } \sim M, \quad N_{1} \text { nicht } \sim N
$$

und damit ist in der Tat die behauptete Nichtäquivalenz eine Folge von (II) und (III). Also

11. Für endliche (und fremde) Mengen $M$ und $N$ gilt die Beziehung

$$
\left(\boldsymbol{M}_{1}, \boldsymbol{N}\right) \boldsymbol{b}(\boldsymbol{M}, \boldsymbol{N}) \text {. }
$$

12. Die Verbindungsmenge zweies endlichen Mengen ist selbst endlich; d. h. aus $M d M$ und $N d N$ folgt $(M, N) d(M, N)$.

Wir haben nachzuweisen, daß die Beziehung

$$
(M, N) a(M, N)
$$

ausgeschlossen ist. Nun hat jede Teilmenge von $(M, N)$ wieder eine der Formen

$$
M, M_{1}, N, N_{1},\left(M, N_{1}\right),\left(M_{1}, N\right),\left(M_{1}, N_{1}\right)
$$

und wir beweisen, genau wie eben (vgl. anch $\$ 5,2$ ), daß keine dieser Mengen zu $(\boldsymbol{M}, \boldsymbol{N})$ äquivalent ist. Damit ist der Satz bewiesen.

\section{$\S 5$.}

\section{Das Äquivalenzproblem.}

Die wichtigste Aufgabe, die zu behandeln ist, betrifft den Nachweis, daß die Mengen $M$ ind $\boldsymbol{N}$ äquivalent sind, falls für sie die Beziehung

$$
M a N \text { oder } M d N
$$

besteht; also der Satz (Aquivalenzsstz)

1. Aus $M a N$ oder $M d N$ folgt $M \sim N$.

Ehe der Beweis geführt wird, sollen die Äquivalenz-Relationen vorangestellt werden, die sich aus den vorstehenden Paragraphen unmittelbar ergeben:

2. Aus $M b N$ und $M a N$ folgt $M$ nicht $\sim N$.

Wäre nämlich $M \sim N$, so hätte man auch $(\$ 3,12)$

$$
N b N \text { oder } N C N \text {, }
$$


was abèr gemäß $\$ 3,3$ widerspruchsvoll ist. Hieraus folgt unmittelbar weiter

3. Mit $M \sim N$ ist mur $M a N$ oder $M d N$ verträglich.

Die Umkehrung dieses Satzes 3 ist es, die den eigentlichen Äquivalenzsatz 1 bildet. Ist er bewriesen, so folgt endlich noch, als Umkehrung von 2.:

4. Aus $M$ nicht $\sim N$ folgt $M b N$ oder $M c N$.

Man kann diese vier Sätze auch folgendermaßen żusammenfassen: Die Beziehungen (a) und (d) sind hinreichende und notwendige Bedingungen für die Aequivalenz, (b) und (c) ebenso für die Nichtäquivalenz. muBte,

Als Folge von 4. ergibt sich, was in $\$ 3$ und 4 noch offen bleiben

5. Aus $M_{2}$ t M und $M_{1}$ nicht $\sim M$ folgt $M_{1} b M ;$ d. h. besteht für die Teilmenge, $M_{1}$ von $M$ die Bexiehung $M_{1}$ nicht $\sim M$, so gilt $M_{1} b M$.

Denn nach 4. gilt $M_{1} b M$ oder $M_{1} c M$; nach Satz 13 von $\$ 3$ nur $M_{1} a M$ oder $M_{1} b M$, also gilt $M_{1} b M$.

Eine Anwendung hiervon gibt auch Antwort auf die bezïglich des Axioms II in \$4 gestellte Frage. Is folgt jetzt

6. Sind $M_{1}$ und $N_{1}$ Teilmengen von $M$ und $N$, und ist $M_{1}$ nicht $\sim M, N_{1}$ nicht $\sim N$, so folgt daraus stets $\left(M_{1}, N_{1}\right) b(M, N)$.

Wir gehen num zum Satz 1 über und beweisen zunächst den ersten Teil, also den eigentlichen Bernsteinschen Äquivalenzsatz. Sein Beweis folgt aus dem Axiom II von-\$4 über die Nichtäquivalenz der Verbindungsmengen.

Aus der Voraussetzung $M a N$ folgt zunächst

$$
\text { ein } M^{\prime} \sim N, \text { ein } N^{\prime} \sim M .
$$

Wäre nun $M$ nicht $\sim N$, so hätte man nach $\$ 1,3$

$$
M \text { nicht } \sim M^{\prime}, N^{\prime} \text { nicht } \sim N \text {. }
$$

Mit $M$ und $N$ sind aber auch $M^{\prime}$ and $N^{\prime}$ fremde Mengen $(\$ 2,1)$; sio bestimmen daher eine Menge $\left(M^{\prime}, N^{\prime}\right)$, und für sie müßte gemäß Axiom II nunmehr

$$
\left(M^{\prime}, N^{\prime}\right) \text { nicht } \sim(M, N)
$$

folgen. Andererseits folgt aber aus den beiden ersten Relationen unmittelbar nach \$ 4, I

$$
\left(M^{\prime}, N^{\prime}\right) \sim(M, N)
$$

und damit ergibt sich eix Widerspruch. Damit ist der Beweis bereits geliefert.

Freilich beruht der Beweis auf einer gewissen Voranssetzung, die noch 
zu exörtern ist. Wir operieren mit der Verbindungsmenge von $M$ und $N$ und haben deshalb die Voraussetzung nötig, $\mathrm{daB} M$ und $N$ fremde Mengen sind. Sind sie es nicht, so wird man am einfachsten so vorgehen, da $B$ man folgendes neue Axiom zugrunde legt ${ }^{13}$ ):

I. Sind $M$ und $N$ keine fremden Mengen, so gibt es stets zwei ihnen äquivalente, zueinander fremde $M$ Mengen $\mathfrak{M}$ und $\mathfrak{R} ;$ so da $\beta$ also

$$
\mathfrak{M} \sim M \text { und } \mathfrak{R} \sim N \text {, und } \mathfrak{M f} \mathfrak{R} \text {. }
$$

Gemäß \& 3,12 besteht auch für sie die Beziehung

$\mathfrak{M} a \mathfrak{R}$,

und auf sie läßt sich daher der obige Beweis übertragen. Aus $\mathfrak{M} \sim \Re$ folgt dann auch $M \sim N$.

Es handelt sich nun noch um den gleichen Nachweis für die Beziehung $M d N$. Ehe ich dazu übergehe, erinnere ich daran, daß die Eigenart der Beziehung $M d N$ in der Cantorschen Theorie offen geblieben war; fïr das durch sie bedingte Verhältnis von $M \mathrm{zu} N$ hatte sich ein Resultat nicht ableiten lassen. Das darf nicht wundernehmen; das hierin enthaltene Problem stellt nämlich wieder ein logisch unlösbares Problem, und damit eine illusorische Aufgabe dar. Wir haben ja als Prämissen zunächst nur die Aussagen

$$
\text { kein } M_{1} \sim N, \text { kein } N_{1} \sim M \text {. }
$$

Dazu kommen, da $M$ und $N$ endliehe Mengen sind,

$$
\text { kein } M_{1} \sim M, \quad \text { kein } N_{1} \sim N,
$$

also lauter Aussagen von negativem Charakter. Selbst der Weg des indirekten Beweises ändert daran in diesem Fall nichts; denn man müBte noch die Annahme

$$
\text { . M nicht } \sim N
$$

hinzufïgen. Nun wäre es ja möglich, daB die für den Beweis einzig in Frage kommenden Axiome II und III der Nichtäquivalenz von $\$ 4$ die Prämissen positiv beeĭnflussen könnten; aber anch das ist nicht der Fall. Denn diese Axiome lauten $j a$ in ihrem SchluBteil übereinstimmend

$$
\left(M_{1}, N_{1}\right) \text { nicht } \sim(M, N) \text {. }
$$

Wir müssen also von Prämissen ausgehen, die samt und sonders negativ sind, und kommen zu dem Schluß, daß sich die Aquivalenz $M \sim N$ im Fall endlicher Menge ohne eine nochmalige neue axiomatische Fest-

13) Es entspricht dem von Zermelo in seinen Grandiagen (Math. Ann. 65) enthaltenen Theorem 19. Man könnte auch die gxößte gemeinsame Teilmenge von $\boldsymbol{M}$ und $N$ axiomatisch einfübren und mit ihr operieren; doch seheint dies weniger einfach. 
setzang nicht folgern läBt. Das so gewonnene Resultat läBt sich auch in seiner allgemeinen Bedeutung leicht verstehen. Es läuft dem Tatbestand parallel, der uns ans der allgemeinen Theorie der endlichen Zahlgrößen geläufig ist. Dort muß die Festsetzung, wann zwei GröBen als gleich gelten sollen, erst frei - natürlich zweckmäßig - geformt werden, ehe man die Frage, $o b$ zwei gegebene GröBen als gleich za gelten haben, in Betracht ziehen kann. Man denke z. B. an die WeierstraBsohe Theorie der Irrationalzahlen; sie setzt bekanntlich die Gleichheit zweier Zahlen $a$ und $b$ so fest, daB jeder Bestandteil von $a$ kleiner ist als $b$ und jeder Bestandteil von $b$ kleiner als $a$. Eine solche exiomatische Festsetzring erweist sich also anch im Gebiet der endlichen Mengen, wenn man sie, wie hier, ausschließlich auf die Mengenbeziehungen, d. h. auf die Nichtäquivalenz von Menge und Teilmenge gründet, als eine Notwendigkeit.

Es fragt sich nur, wrelche Festsetzung man zweckmäBig zugrunde legt. Beachtet man, daß es sich im Grunde um eine Axiomatik der GröBenlehre handelt, so liegt offenbar nichts näher, als die eben genannte Definition zu benutzen, und dies soll in der Tat geschehen. Wir setzen also fest (Axiom der Aquivalenz endlicher Mengen):

II. Zwei endliche Mengen $M$ und $N$ sind äquivalent, wenn für jede Teilmenge $M^{\prime}$ und $N^{\prime}$ die Beziehung $M^{\prime} b N$ resp. $N^{\prime} b M$ besteht; d. h. aus $M d M, N d N, M^{\prime} b N, N^{\prime} b M$ für jedes $M^{\prime}, N^{\prime}$ folgt $M \sim N$.

Hieraus läßt sich der Satz, daB aus $M d N$ auch $M \sim N$ folgt, unmittelbar folgern. Ehe wir dazu übergehen, wollen wir noch die Berechtigung unseres Axioms und seine Stellung im gessmten Aufbau näher erörtern. Wir wollen zunächst nachweisen, daB von den vier Beziehungen

$$
M a N, M b N, M c N, M d N \text {. }
$$

nur die betzte mit dem Axiom verträglich ist.

Aus $\boldsymbol{M a} \boldsymbol{N}$ folgt

$$
\text { ein } M^{\prime} \sim N
$$

gemäB unserm Axiom ist aber für jedes $M^{\prime}$

$$
\boldsymbol{M}^{\prime} b \boldsymbol{N} \text {, }
$$

und man exhielte also $N b N$, was aber nach $\$ 3,3$ widerspruchsvoll ist.

Aus $M b N$ folgt

$$
\operatorname{ein} N^{\prime} \sim M
$$

was anslog zur Relation $M b M$ führt, die ebenfalls widersprachsvoll ist. Bndlich folgt ans $M c N$ genau wie eben die widerspruchsvolle Relation $N B N$.

Unser Axiom kann gląso in der Tat nur "mit der Béziehung $M d N$ 
verträglich sein. Dies ist aber auch wirklich der Fall. Die Folgerungen, die sich aus

$M^{\prime} b N$ und $M d N$, aus $N^{\prime} b M$ und $M d N$

ergeben, lauten gemäß $\S 3,9$, daß für jedes $M^{\prime}$ und $N^{\prime}$

$$
M^{\prime} b M \text { und } N^{\prime} b N
$$

ist; sie entsprechen der Endlichkeit von $M$ und $N$ und stellen die in $\$ 4,4$ gefundene Eigenschaft der endlichen Mengen dar.

Zusammenfassend folgt also: Das Axiom II ist nur für endliche Mengen realisiert, und überdies weder im Fall $M b N$, noch $M c N$; damit ist aber der Beweis seiner Berechtigung geliefert. Ess ist für die endlichen Mengen und ihre Aquivalenz charakteristisch.

Der Beweis des Aquivalenzsatzes ergibt sich nun folgendermaßen.

GemäB \& 4, Satz 4 ist für jedes $M^{\prime}$ und $N^{\prime}$

$$
M^{\prime} b M \text { und } N^{\prime} b N \text {; }
$$

ferner gilt nach Voraussetzung

$$
M d N \text { und } N d M \text {, }
$$

und hieraus folgt nach $\$ 3,9$ sofort

$$
M^{\prime} b N \text { und } N^{\prime} b M
$$

und nunmehr. nach unserm Axiom

$$
M \sim N
$$

$\$ 6$.

\section{Sätze über Verbindungsmengen.}

Seien $M$ und $N$ einerseits, und $\mathfrak{M}$ und $\mathfrak{R}$ andrerseits fremde Mengen. Zwischen $M$ und $\mathfrak{M}$, sowie zwischen $N$ und $\mathfrak{R}$ bestehe je eine der $\mathrm{Be}$ ziehungen

und

$$
\begin{aligned}
& M a \mathfrak{M}, \quad M b \mathfrak{M}, \quad M c \mathfrak{M}, \quad M d \mathfrak{M} \\
& N a \Re, \quad N b \Re, \quad N c \Re, \quad N d \Re \text {. }
\end{aligned}
$$

Ers ist die Frage, welche Beziehung für

$$
(M, N) \text { and }(\mathfrak{M}, \mathfrak{R})
$$

resaltiert, wenn wir irgendeine Beziehnng der ersten Zeile mit einer Beziehung der zwriten Zeile kombinieren.

Wix beweisen zunächst folgende sätze:

1. Aus $M a \mathfrak{M}$ und $N a \mathfrak{R}$ folgt $(M, N) a(\mathfrak{M}, \mathfrak{N})$.

2. Aus $M b \mathfrak{M}$ and $N b \Re$ folgt $(M, N) b(\mathfrak{R}, \mathfrak{R})$.

3. Aus $M \subset \mathfrak{M}$ und $N \subset \mathfrak{R}$ folgt $(M, N) \subset(\mathfrak{M}, \mathfrak{R})$.

4. Aus $M d \mathfrak{M}$ und $N d \mathfrak{R}$ folgt $(M, N) d(\mathfrak{M}, \mathfrak{R})$.

5. Aus $M a \mathfrak{R}$ und $N d \Re$ folgt $(M, N) a(\mathfrak{M}, \mathfrak{R})$. 
Die Beweise von Satz 1, 4, 5 lassen sich folgendermaßen zusammenfassen. Die Voraussetzungen lauten gemeinsam

$$
M \sim \mathfrak{M} \text { und } N \sim \mathfrak{N} \text {, }
$$

woraus gemäß Axiom $I$ von $\$ 4$

$$
(\boldsymbol{M}, N) \sim(\mathfrak{M}, \mathfrak{R})
$$

folgt. Im Fall 1 und 5 sind nun $M$ und $\mathfrak{B}$ nach $\S 4$, Satz 3 anendliche Mengen, also gilt dies nach $\$ 4,8$ auch von $(\mathcal{M}, N)$ und $(\mathfrak{M}, \mathfrak{M})$ and daher exgibt sich wieder

$$
(\boldsymbol{M}, \boldsymbol{N}) \boldsymbol{a}(\mathfrak{M}, \mathfrak{R}) \text {. }
$$

Im Fall 4 sind dagegen $M, N, \mathfrak{N} R, \mathfrak{A}$ endliche Mengen, also auch $(\$ 4,12)(M, N)$ und $(\mathfrak{M}, \mathfrak{R})$ und daher ist

$$
(\boldsymbol{M}, \boldsymbol{N}) d(\mathfrak{M}, \mathfrak{R}) \text {. }
$$

Wir beweisen nun den Satz $\mathbf{2}^{14}$ ). Dazu gehen wir von den Relationen $M b M$ und $N b \Re$

aus, also von den Beziehungen

$$
\begin{array}{ll}
\text { kein } M_{1} \sim \mathfrak{M} & \mathfrak{M}^{\prime} \sim M, \\
\text { kein } N_{1} \sim \mathfrak{N} & \mathfrak{N}^{\prime} \sim N,
\end{array}
$$

und erhalten zunächst

$$
\left(\mathfrak{M} \mathbb{R}^{\prime}, \mathfrak{N}^{\prime}\right) \sim(\boldsymbol{M}, N) .
$$

Wir folgern nun aus den gegebenen Relationen $M b M$ und $N b \Re$ mittels $M \sim \mathfrak{M}^{\prime}$ und $N \sim \mathfrak{R}^{\prime}$ weiter

oder aber $(\$ 5,2)$

$$
\mathfrak{M} b \mathfrak{M} \text { und } \mathfrak{Z}^{\prime} b \mathfrak{R}
$$

$$
\mathfrak{M R}^{\prime} \text { nicht } \sim \mathfrak{M}, \quad \mathfrak{R}^{\prime} \text { nicht } \sim \mathfrak{R}
$$

and daraus endlich, gemäß Satz 6 von $\$ 5$

$$
\left(\mathfrak{M} \mathfrak{R}^{\prime}, \mathfrak{R}^{\prime}\right) b(\mathfrak{M}, \mathfrak{R})
$$

oder

$$
(\mathcal{M}, N) b(\mathfrak{N}, \mathfrak{R}) \text {. }
$$

14) Goht man zu Mrohtigkeiten über, so bezieht sich der obige Satz auf den Fall, daB

"ito; er sohliebt daraus

$$
\begin{gathered}
\mathfrak{m}_{1}<m_{4} \text { und } n_{2}<n_{3} \\
m_{1}+n_{1}<m_{2}+n_{2} .
\end{gathered}
$$

In der allgemeinen Theorie fehlt noch heute ein Nachweis dieser Folgerung. Sie ist von $F$. Bernetein unter der Annahmo bewiesen worden, daß gleichbar" ist. (Math. An. 61 (1905), S. 129.) Nun scheidet zwar in dem vorliegenden Aufbar die Vergleichbarkeit als offene Prage gemäf \& 3 aus, der Berneteinsche

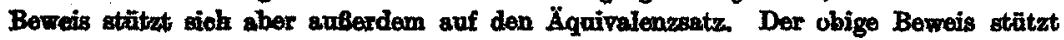
wich dagogen anf das Axiom $\mathbf{I}$ von \& 4, das ja anch den Barnsteingohen Aquivelonzcone Folgo hat. 
In derselben Weise beweist man den Satz 3. Ein letzter Sakz, der sich ableiten läbt, lautèt:

6. Ișt $M$ eine endliche Menge, so folgt aus $M b \mathfrak{M}$ und $N d \mathfrak{R}$

$$
(\boldsymbol{M}, \boldsymbol{N}) \boldsymbol{b}(\mathfrak{M}, \mathfrak{R}) \text {. }
$$

Wegen $M b \mathfrak{R}$ hat man nämlich

$$
M \sim \mathfrak{M}^{\prime}
$$

wo mit $M$ auch $\mathfrak{M}^{\prime}$ eine endliche Menge ist. Hieraus und aus $N \sim \mathfrak{R}$ folgt weiter

$$
(M, N) \sim\left(\mathfrak{M}^{\prime} \mathfrak{R}\right)
$$

Wir unterscheiden nun, ob $\mathfrak{M}$ eine endliche oder unendliche Menge ist. Im ersten Fall sind $\left(\mathfrak{M}^{\prime}, \mathfrak{R}\right)$ und $(\mathfrak{M}, \mathfrak{R})$ endliehe Mengen, ferner ist $\left(\mathfrak{M}^{\prime}, \mathfrak{N}\right)$ Teilmenge von $(\mathfrak{M}, \mathfrak{N})$ und deher ist gemäß $\S 4,4$

$$
\left(\mathfrak{M}^{\prime} \mathfrak{R}\right) \boldsymbol{b}(\mathfrak{M} \mathfrak{R}) \text {. }
$$

Ist aber $\mathfrak{M}$ eine unendliche Menge, so ist $(\mathfrak{M}, \mathfrak{R})$ nach $\S 4,8$ ebenfalls eine unendliche Menge; dagegen ist $(\mathfrak{M}$ ', $\mathfrak{R})$ nach $\S 4,12$ endlich und daher gilt ebenfalls $(\$ 4,7)$

$$
\left(\mathfrak{M} \mathfrak{R}^{\prime}, \mathfrak{N}\right) \boldsymbol{b}(\mathfrak{M}, \overline{\mathfrak{R}}) \text {. }
$$

Wegen $\mathfrak{M} \mathfrak{R}^{\prime} \sim M, \mathfrak{M} \sim \boldsymbol{N}$ folgt daraus weiter

$$
(M, N) b(\mathfrak{M}, \mathfrak{R}) \text {. }
$$

In den anderen Fällen lassen sich eindeutige Folgerungen nicht entnehmen. Nur soviel sei bemerkt, daß mit den Relationen

jede der beiden Beziehungen

$$
M a \mathfrak{R} \text { und } N b \mathfrak{R}
$$

verträglich ist.

$$
(M, N) a(\mathfrak{R}, \mathfrak{R}) \text { und }(M, N) b(\mathfrak{R}, \mathfrak{R})
$$

\section{\& 7.}

\section{Schlugbetrachtung.}

Die vorstehende Untersuchung liefert jedenfalls ein hinreichendes Axiomensystem für die Sätze, die die Åquivalenzprobleme der Mengen betreffen. Wird für den Augenblick noch die Bezeichnung $-M e N$ für die Aquivalenz von $M$ und $N$ eingeführt, so handelt es sich, genauer gesprochen, um die Kombination der Beziebungen, die durch

$$
M a N, M b N, M c N, M d N, M B N, M f N, M t N,(M, N)
$$

dargestellt sind, und um die Art, wie sie assoziativ einander bédingen und 
sich miteinander verbinden. $\mathrm{Ob}$ die aufgestellten Axiome sämtlich notwendig sind oder auch entbehrliche Bestandteile enthalten, mag offen bleiben. Abgesehen von den Axiomen mehr formaler Bedeutung, wie die über $M \in N, M f N, M t N$ sind es wesentlich die folgenden, die die materiellen Stützen des Aufbaues darstellen: Das Axiom der Verknüpfung, die Axiome über die Aquivalenz der Teilmengen und der Verbindungsmengen, die Axiome über die Nichtäquivalenz der Verbindungsmengen nicht äquivalenter Mengen und das Axiom über die Aquivalenz endlicher Mengen. Die Charakterisierung, die in diesen Bezeichnungen enthalten ist, zeigt schon die Verschiedenheit der Gebiete, denen sie angehören, und zeigt auch ihre allgemeine Notwendigkeit für den Aufbau.

Wie bereits in der Einleitung erwähnt, ist die vorstehende Betrachtung zugleich eine Axiomatik der Größenlehre; in der Tat ist ja von den Elementen der Menge nirgends die Rede. Dies ist anch die Tatsache, die dem in $\$ 3$ gefundenen Resultat seine Stellung im axiomatischen Aufbau anweist. Wir fanden dort, $d a B$ mit den Beziehungen $M d N$ und $N d P$ auch die Folgerung $M a P$ verträglich ist. Sie könnte deshalb an sich ebenfalls als axiomatische Festsetzung an Stelle des Axioms II eingeführt werden. Wie wir sahen, bewirkt sie als weitere Folgerung, daß aus $M$ a $P$ and $P d N$ sich $M d N$ ergibt, and liefert ebenfalls ein in sich widerspruchsfreies System von Beziehungen. Es ließ sich durch die Formeln

daratellen.

$$
(a a)=(d d)=a ; \quad(a d)=(d a)=d
$$

Dies wollen wir nun denten. Zunächst ist za beachten, daB in die vorstehenden Schlïsse die Beziehungen $M b N$ und $M c N$ nicht eingehen. daß es sich bei ibnen vielmehr nur um $M a N$ und $M d N$ und deren Kombinationen handelt. Nur auf sie beziehen sich also die obigen Regeln, und auf sie beschränke ich mich zunächst. Die Aufgabe ist dann, Objekte mit GröBencharakter zu finden, die sich diesen Regeln fügen. Die in \$ 3 erwähnte Analogie mit den Vorzeichenregeln macht dies leicht. Man erreicht es, indem man entgegengesetzte Größen in Betracht zieht, deren Teile zum Ganzen in der dureh (a) festgelegten Bexiehung stehen, also dex Dedekindschen Definition genügen; die Beziehung $M a N$ gilt dann für gleichartige, dagegen $\boldsymbol{M} d \boldsymbol{N}$ für entgegengesetzte Objekte. Finseitig begrenzte Geraden von unendlicher Länge aber entgegengesetzter Richtung bilden ein einfaches Beispiel, falls man als Teilmenge jeden ebenfalls unendlichen Bestandteil betrachtet und die Aquivalenz z. B. durch eineindeutige Ahnlichkeitsabbildang definiert. Für je zwei von ihnen besteht dann entweder die Relation (a) oder (d).

Man' kann leicht "erreichen; daB auch die Beziehnngen (b) und (c) auftreten. Dies geschieht sa, daB man auch Paare entgegengesetat gerich- 
teter Geraden als Objekte zuläBt. Für je zwei solehe Parre besteht dann die Beziehung ( $a$ ), für jedes Parar und eine einzelne Gerade die Beziehung (b) oder (c), und für je zwei einzelne Geraden die Beziehung $(a)$ oder $(d)$. Die Gesetze

$$
(a a)=(d d)=a, \quad(a d)=(d a)=d
$$

bleiben offenbar bestehen. Bexiehungen $(b b),(b d),(d c)$ und $(c c)$ sind unmöglich. Dagegen gibt es hier eine Regel für $(b c)$; es kann sowohl $(a)$ wie $(d)$ resultieren. Endlich ergeben die Beziehungen

$$
(a b)(b a)(d b), \quad(a c)(c a)(c d)
$$

(b) oder (c) als Resultat.

Die Tatsache, dab die Cantorsche Theorie die Unvereinbarkeit der Annahme, $M$ und $N$ seien unendliche Mengen, mit der Beziehrng $M d N$ des $\$ 3$ nicht nachzuweisen vermochte, erfährt hierdurch nenes licht. Denn die Zulassung von Elementen von zweierlei Art, die einander entgegengesetzt sind, streitet weder gegen den Mengenbegriff als solchen, noch auch gegen die Dedekindsche Definition der unendlichen Mengen und die auf ihr ruhenden Eigenschaften. Für den so erweiterten Mengenbegriff kann aber, wie wir sahen, im Fall unendlicher Mengen auch die Beziehung $M d N$ realisiert sein. Wie weit sich auf solche Mengen die weiteren Begriffe und Sätze der Cantorschen Theorie übertragen lassen, mag an dieser Stelle auf sich beruhen bleiben ${ }^{15}$ ).

Nux das sei noch erwähnt, daß die allgemeine Weiterführung der bisher gefundenen Resultate in erster Linie die Beziehung der Menge zu ibren Elementen, ferner den Ordnungsbegriff usw. ins Auge zu fassen hat. Ich will noch kurz zeigen, wie man die Elemente der Menge auf der hier vorhandenen Grundlage einfühihen kann. Voranzustellen ist das folgende Axiom:

1. Jede Menge enthält Teilmengen, die nicht mehr selbst in Teilmengen zerlegbar sind; sie heißen unzerlegbare Teilmengen oder Elemente. Sie sollen durch

$$
m T M \text { oder kürzer durch } m
$$

bezeichnet werden. Von ihnen gilt der Satz:

Ist $M \sim N$, so kann eine nicht zerlegbare Teilmenge von $M$ keiner zerlegbaren Teilmenge von $N$ äquivalent sein und umgekehrt.

Aus der Aquivalenz $M \sim N$ folgt nämlich nach Axiom I von \$3 zu jedem $M^{\prime}$ die Existenz einer Teilmenge $N^{\prime}$ von $N$, so daß

$$
M^{\prime} \sim N^{\prime}
$$

19) Herr A. Fränkel hat mich daranf hingawiesen, daB nioht einmal alle Axiome über Teilmongen gelten. Axiom I bleibt bestehen, Axiom II nicht; es gilt auch Axiom III von \& 8. 
ist. Würde nun $m=M^{\prime}$ ein zerlegbares $N^{\prime}$ bedingen und wäre $N^{\prime \prime}$ eine Teilmenge von $N^{\prime}$, so folgt aus $M^{\prime} \sim N^{\prime}$ gemäB demselben Axiom, daß $N^{\prime \prime}$ die Existenz einer Teilmenge von $m$ bedingt, die $z \mathrm{u} N^{\prime \prime}$ äquivalent ist; was aber einen Widerspruch darstellt.

Von diesem Tatbestand kann man nun wieder verlangen, $d a B$ er auch umgekehrt gilt; d. h. man kann fordern:

II. Zwei Mengen $M$ und $N$ sind äquivalent, wenn jedem Element von $M$ ein Element von $N$ zugehört und umgekehrt.

$\mathrm{DaB}$ diese Forderung an sich widerspruchstrei ist, wurde eben gezeigt; daß sie anch den allgemeinen Axiomen genügt, die die Äquivalenzbeziehung regeln $(\S 1, \mathrm{I}$ und II, $\S 3, \mathrm{I}, \S 4, \mathrm{I})$, ist leicht zu sehen. Damit möge diese Betrachtung ihren Abschluß finden. Auf die Frage, wie mit der Einfïhrung der Elemente und der neuen Äquivalenzbeziehung sich der axiomatische Aufban ändern wiirde, soll hier nicht weiter eingegangen werden.

Jedenfalls entspricht die vorstehende Untersuchung den Forderungen, die im Anfang gestellt wurden. Sie sieht von allen Wortdefinitionen $a b$ und benutzt ausschlieBlich Beziehungen zwischen den Objekten, von denen sie handelt. Die Axiome liefern die Grundregeln für das Operieren mit ihnen. Gerade um dies deutlich hervortreten zu lassen, ist jedem Axiom und jedem Satz die ihm entsprechende formale Ausdrucksweise, also die Bindung, die die bezüglichen Beziehungen durch den Satz oder dás Axiom erfahren, gegeben worden. Auch sind die einzelnen Axiome immer erst dann eingeführt worden, wenn sie für den Fortgang der Beweise nötig waren.

(Fingegangen am 14. 12. 1920.) 